\title{
LSS Implementation in Micro Enterprises: Adoption of Tools to Support Competitiveness
}

\author{
Seamus O’Reillya (s.oreilly@ucc.ie), \\ Dermot Freeman ${ }^{b}$ (info@dermotfreeman.com) \\ and Lawrence Dooley ${ }^{a}$ (l.dooley@ucc.ie) \\ ${ }^{a}$ Cork University Business School, Ireland. ${ }^{b}$ Dermot Freeman \& Associates, Ireland
}

\section{Purpose}

This study seeks to contribute to an emerging literature addressing Lean Six Sigma (LSS) implementation in the micro enterprise context. Specifically, it explores what LSS tools address business opportunities and challenges identified by micro enterprises.

\section{Methodology}

This study adopts a purposive sampling approach. Thirty micro enterprise who had availed of a lean start programme launched by a public enterprise support agency were surveyed. A telephone survey followed a semi-structured interview format. This approach resulted in good participation rate and facilitated a conversation to establish motivation, initial use and impact of LSS tools and a discussion on the ongoing use of tools within these enterprises.

\section{Findings}

Time management and the need to improve organisational structures emerged as the main challenges faced by owner-managers. The introduction of a LSS mindset and use of tools built a new capability within these enterprises to address these challenges. The ownermanagers were internally focused on structure and systems, internal process improvement and efficiency identified as main reasons to introduce LSS tools

\section{Limitations}

This is an exploratory survey of 30 micro enterprises. Additional observations would enhance our understanding of micro enterprise implementation of LSS.

\section{Originality}

While use of LSS tools by SMEs has received some attention in recent years, this is an area that merits further research. In addition, micro enterprises have received very little attention, given their specific organisational context this study focuses on these enterprises.

\section{Practical Implications}

The study provides practical insight into how micro enterprises may use LSS tools to improve competitiveness, in particular the benefits accruing from improved organisational structures and processes. It also suggests further research that would inform both policy makers and contribute to understanding of the potential of LSS in a micro enterprise environment.

Keywords: Lean Six Sigma, micro enterprises, LSS tools, SMEs.

Article Classification: Research Paper 


\section{Introduction}

Signoretti (2020) argues that rigorous continuous improvement tools and specific managerial principles are increasingly important to support SME competitiveness in relation to costs and reliability (lead-time and quality). However, a body of literature points to barriers to lean/six sigma implementation in SMEs (Alkhoraif et al., 2019). This study seeks to contribute to an emerging literature addressing Lean Six Sigma (LSS) implementation in the micro enterprise context. Specifically, it aims to explore what LSS tools and approaches support micro enterprise competitiveness.

\section{Literature Review}

Alkhoraif et al., (2019) highlighted a lack of attention to lean implementation in SMEs compared to a more developed knowledge base within larger enterprises. Their review and analysis points to both internal and external supply chain aspects of lean, finding that these are well developed capabilities in larger enterprises compared to SMES. Indeed, they conclude that "the size of the firm would influence lean implementation, in both a company and its supply-chain. The current standpoint of literature suggests that, for the most part, only larger companies have implemented Lean and done so successfully" (p.14). Similarly, Panayiotu and Stergio (2021), on the basis of a systematic literature review of LSS projects in large organisations and SMES, call for analysis of the factors that facilitate LSS adoption in SMEs.

In looking to critical success factors (CSF) that support LSS implementation a considerable body of literature based on comprehensive reviews and research has developed, for example, Cornodo and Antony (2002), Achanga, et al., (2006), Naslund (2013) and Albliwi et al., (2014). Adopting a CSF approach Cornodo and Antony (2002) identified the key ingredients of successful deployment of Six Sigma. This foundational work highlighted the role of management in terms of leadership commitment, organisational culture and communication, linkage with human resources, development of skills and organisational capability. This work also identified the supply chain context drawing attention to the need to start with customer requirements and deploy Six Sigma techniques to identify and deliver critical-to-quality characteristics (CTQs) and recognise the need to work with suppliers not only from a cost reduction perspective but also quality through a Six Sigma capability to address variance. Exploring and addressing the complementary nature of Lean and Six Sigma, Laureani and Antony (2012) identified CSFs for effective implementation of Lean Six Sigma (LSS). This research supported earlier work and highlighted the need to align LSS with strategy, the need for appropriate leadership styles, skills and capability development and extending LSS to the supply chain. These CSFs are reflected in the body of literature that has contributed to our understanding of LSS implementation over recent years (Alkhoraif, et al., 2019).

When addressing SME implementation of LSS it is informative to look to this foundational literature and consider applicability to the SME context. Indeed, a recent systematic review of literature on LSS deployment by manufacturing SMEs has drawn usefully on the knowledge built on CSFs for LSS implementation more generally (Alexander, et al., 2019). Our study benefits from this work and considers the findings in the context of this CSF 
literature and seeks to contribute to an emerging literature on CSFs deployment by micro, small and medium size enterprises.

\section{Context}

There has been considerable activity amongst micro, small and medium enterprises in Ireland in the LSS arena. This is largely due to support provided by Irish enterprise support agencies over the last 15 years. For example, Enterprise Ireland's lean business offer included a comprehensive range of supports, from introduction to concepts and tools (LeanStart), through "process improvement" (LeanPlus) and ultimately building "organisational innovation" capacity (LeanTransform) ${ }^{1}$. Various reports indicate active engagement by industry, including SMEs. Enterprise Ireland (April 2015), reports 343 companies participating between 2009 and 2012, with majority in the SME category. Given this level of engagement public agencies tasked with supporting micro-scale enterprises launched a lean start programme in 2015. This programme also attracted a high level of engagement with 950 micro enterprise lean start projects completed in 2019. This policy driven activity presents an interesting micro enterprise population for research, exploring their experiences and, in particular, the link between motivation to engage in continuous improvement and choice of LSS tools, and level of success achieved. Thus, this study aims to contribute to the literature and inform enterprise support policy.

\section{Methodology}

A purposive sampling approach was adopted. A population of thirty-five micro enterprises that had availed of public support agency support to introduce continuous improvement, during the period 2017-2019, were randomly selected. The EC (2003) definition of micro enterprise was used, i.e. $<10$ employees and $\leq € 2 \mathrm{~m}$ turnover. Contact was made by telephone to request participation in the survey (30 of the 35 enterprises approached found the time to participate) and this was followed by an interview or another time was agreed for this. Across the sample there was a high level of activity in the service sector, including retail services, distribution and specialist services (such as construction, film and print). In total $77 \%$ of the sample were involved in services with most of the remainder in manufacturing.

The interview followed a semi-structured format, beginning with an open question as to the motivation to introduce continuous improvement. This facilitated unpromoted discussion that allowed the enterprise owner-manager to convey their business context and underlying reasons that prompted them to avail of public agency support to introduce continuous improvement. These reasons provided insight into key competitive challenges and business opportunities faced by these micro enterprises. A list of LSS tools (informed by Alkhoraif, et $a l ., 2019$ and Alexander et al., 2019) was presented to the respondent to identify and discuss the rational for choice of the selection of lean tools used. An open discussion of the range of tools allowed respondents include additional tools employed. While a list was presented responded were asked to reflect on the tools they employed and, in particular, how successful they have been in initial and ongoing use of these tools. An open discussion facilitated

${ }^{1}$ Lean Business Offer - Enterprise Ireland (enterprise-ireland.com) 
owner-manager reflection on the continuous improvement experience, including impact, and future plans.

\section{Findings}

As the motivation to introduce continuous improvement was explored through open discussion a range of reasons were put forward. These are illustrated in Figure 1.

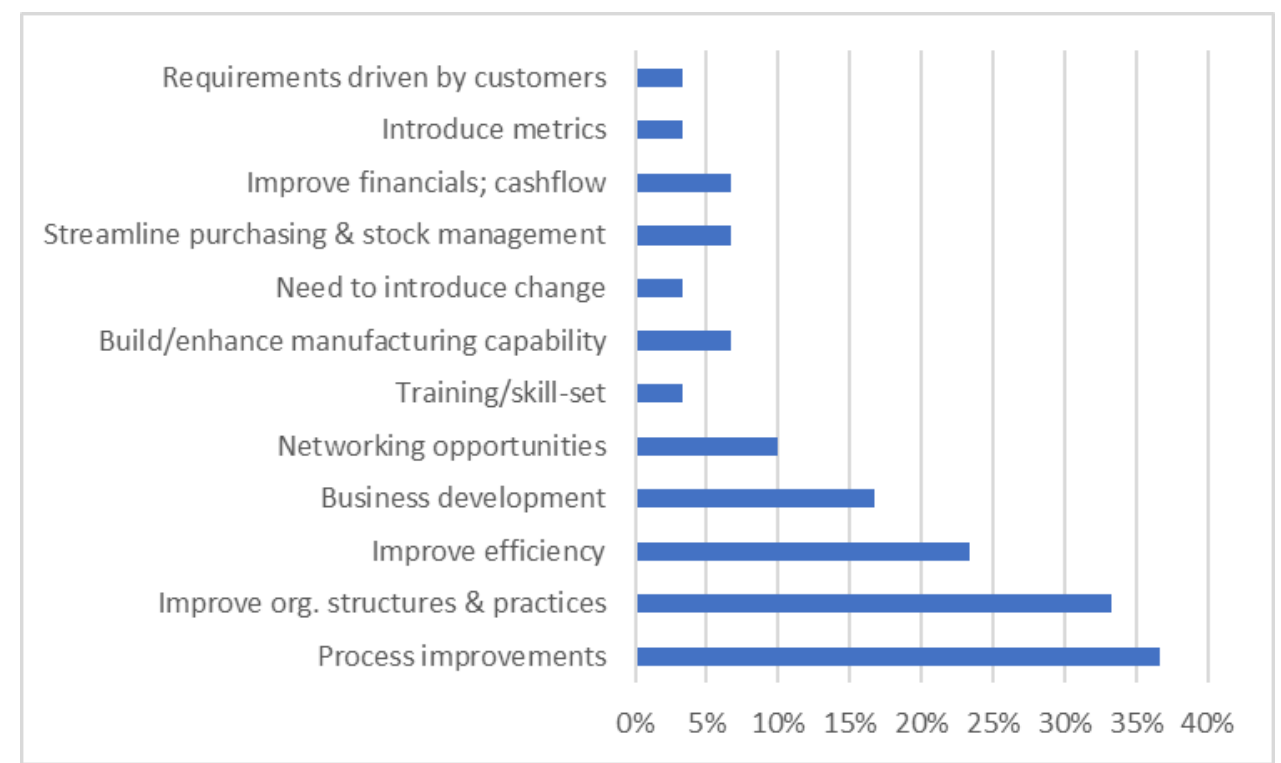

Figure 1: Motivation to introduce Continuous Improvement

The motivations to improve structures, adopt a process-based approach and reduce costs is notable. While the need to improve structure is typical of micro enterprises, it interesting to find that these micro enterprises identify LSS as a means to improve organisational structure. Discussion with respondents revealed that a fundamental driver of this motivation is a lack of time, hence they endeavoured to improve organisational structures and, in some cases, did this to capitalise on growth opportunities. Other than this, the motivations are operational in nature, focused on efficiency and process improvement. Thus, they are internally focused with little attention to the wider supply chain, upstream or downstream. Furthermore, there is little explicit attention to training and metrics, while these are implicit in many of the other reasons, this also raises an interesting question as to the appropriate LSS organisational infrastructure in micro enterprises (e.g. training to various LSS belt levels).

In the main these findings reflect the literature, for example in their review of manufacturing SMEs Alexander et al., (2019) noted an operational focus with reduced cost, improved efficiency and improved quality as benefits. Our findings reflect the focus on cost and efficiency, but little attention was given to quality. Our findings also contrast with those of Alexander et al., (2019) with much greater interest on improving organisational structure. 
They align with Zhou's (2016) findings in the US, where the primary reasons to implement lean were found to be mainly internal, including cost reduction, improve profit margin, improve utilization of plant/facility, and maintain competitive position. Similarly, a systematic review of SME studies by Alkhoraif et al., (2019) found a focus on efficiency, but also on quality which received less attention by the micro enterprise respondents in this study. While quality did not feature strongly as a reason, analysis of use of tools and impact identified process improvement, enhanced organisational structures and overall change which improved quality.

The use of LSS tools and techniques largely reflect the reasons for engaging in Continuous Improvement. Figure 2 presents the tools used and sustained use of these tools. The highlevel of continued use of tools is notable. As reported above, time management emerges as an underlying theme in this study and this, in part, explains the emphasis on improved organisational structures, with respondents expressing; "needed to put structures into the company" (R6); "looking for better ways to do things" (R8); "Looking for systems to streamline purchasing and stock management" (R10); "to install systems to support growth" (R18); "improve structure" (R19) and "improve systems" (R19). Thus while "time management' may not be considered a specific LSS tool it was required by a number of micro enterprises.

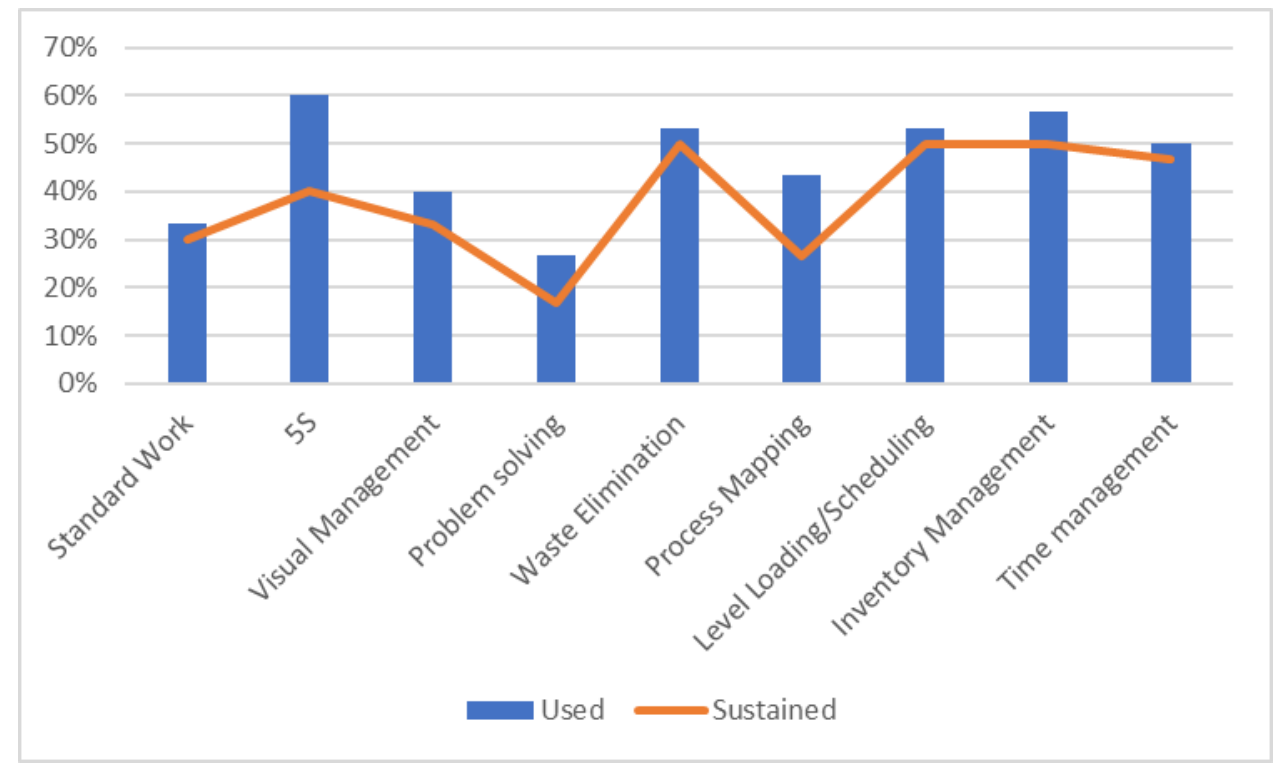

Figure: Use of LSS Tools \& Techniques

The greatest impact of improved structures, process mapping and problem solving was a change in mindset; "Created a mindset that was a different way of thinking" (R4); "was a huge success in that it created a mindset of adding value" (R9); "I believe the Lean mindset has greatly aided the business" (R18). The impact of a mentoring approach adopted by the Lean Expert (Consultant) is most evident; "Mentoring sessions were a major benefit" (R1). Given the established practice of mentoring in micro/small-scale enterprises, adopting this 
approach was key to unlocking the potential of Lean, in that the conversation tended to start from a business problem/opportunity perspective and in this way establish the 'business case' for continuous improvement and identification of appropriate LSS tools.

Over the time period considered, engagement with LSS tools is positive, while two enterprises failed to progress with the initial continuous improvement project the remainder did with positive engagement for most over this period. This in part may be due to the initial dissemination work by enterprise support agencies. Hence, these micro enterprises were already disposed to engagement with continuous improvement. While there is a high level of sustained use of LSS tools in a number of cases enterprises failed to do so. In some cases, this was influence by the COVID-19 Pandemic; "DMB [Daily Management Boards] worked until COVID" (R30). "Was a success in that it created a mindset of adding value - the simple things can make a huge difference, $5 S$ was a success but has relapsed during COVID and warehouse improvements were not maintained" (R9). "Lost the way a little due to staffing during COVID" (R28).

\section{Conclusions and Recommendations}

Alexander et al., (2019) found that lack of time and resources was a common challenge for SMEs, which emerged as fundamental motivation in this study. This influenced the LSS tools adopted but also prompted an internal focus with little attention to the wider supply chain. The predominance of a cost reduction motivation aligns with the use of Lean rather than Six Sigma tools. Should these enterprises grow it is likely that opportunities to reduce variance would become more evident and increase the relevance of Six Sigma tools. However, as the business model of a number of the respondents was based on customisation reducing variance may not be a major issue. Notwithstanding this, as these micro enterprises started to use various tools, the usefulness of Six Sigma tools such as run charts became evident to some. Hence, management and support agencies could explore and address other reasons why micro enterprises may prefer Lean over Six Sigma tools, for example previous research has identified lack of statistical skills and knowledge of Six Sigma as barriers to adoption. As Alexander et al., (2019) caution "being a slave to one or two methodologies can restrict improvement efforts and potential value add" (p. 392). Their advice to avoid a myopic approach and build continuous improvement strategies that adapt to business environment challenges and opportunities through application of appropriate tools could inform policy, such as that pursued in Ireland, and build LSS capability that supports competitiveness over the long term. The benefit of this approach is evident in this study, given the underlying need for time management, including this as a tool together with other LSS tools such as standard work and visual management helped embed Continuous Improvement and sustain benefits.

The initial and ongoing commitment of owner-managers to a continuous improvement philosophy supported ongoing use of and benefit from LSS tools. This reflects the observation by Alexander et al., (2019) that due to their scale senior management commitment tends not to be an issue for SMEs. In this study the business mentoring approach adopted by the consultant was important, as it introduced this philosophy and translated it into projects with clear linkage to owner-manager strategies, i.e. addressing challenges and opportunities. This reflects the leadership commitment and strategy alignment CSFs identified in LSS transformation programmes in larger organisations. In the micro 
enterprises studied the consultant played the role of 'lean expert' and while formal training was conducted it was very much 'hands on' and over a short period of time. Hence, we can see that training, skills and access to a 'lean expert' played a role in the successful introduction of continuous improvement and relevant LSS tools, these CSFs merit attention in future research focused on micro enterprises. Furthermore, while the business mentoring approach emerges as an interesting finding, analysis of the dissemination/animation role of publicly funded enterprise support agencies that introduced these enterprises to continuous improvement approaches were outside the scope of this study and also merit further attention in future research.

This study while exploratory in nature highlights the applicability of LSS tools to micro enterprises. It evaluates the selection and use of LSS tool based on initial motivation and ongoing use of tools. It finds the established CSF LSS literature provide a useful framework for analysis of the introduction of LSS tools in this environment and in doing so points to specific areas that merit further research.

\section{References}

Achanga, P., Shehab, E., Roy, R. and Nelder, G. (2006), "Critical success factors for lean implementation within SMEs", Journal of Manufacturing Technology Management, Vol. 17 No. 4, pp. 460-471.

Albliwi, S., Antony, J., Halim Lim, S.A. and van der Wiele, T. (2014), "Critical failure factors of lean six sigma: a systematic literature review", International Journal of Quality and Reliability Management, Vol. 31 No. 9, pp. 1012-1030.

Alkhoraif, A., Rashid, H. and McLaughlin, P., (2019), "Lean implementation in small and medium enterprises: Literature review", Operations Research Perspectives, 6, p.100089.

Alexander, P., Antony, J. and Rodgers, B. (2019) "Lean Six Sigma for small- and mediumsized manufacturing enterprises: a systematic review", International Journal of Quality \& Reliability Management, Vol. 36 No. 3, 378-397

Cornodo, R. B. and Antony, J. (2002) "Critical Success Factors for the successful implementation of six sigma projects in organisations", The TQM Magazine, Vol. 14. No. 2. 92-99.

EC (2003), Commission Recommendation of 6 May 2003 concerning the definition of micro, small and medium-sized enterprises (Text with EEA relevance) (notified under document number C (2003) 1422)

Enterprise Ireland (2015), Evaluation of Enterprise Ireland Lean Business Offer 2009-2012, Department of Jobs, Enterprise and Innovation, Dublin, Ireland.

Laureani, A. and Antony, J. (2012), "Standards for lean six sigma certification", International Journal of Productivity and Performance Management, Vol. 61 No. 1, pp. 110-120.

Näslund, D. (2013), "Lean and six sigma - critical success factors revisited", International Journal of Quality and Service Sciences, Vol. 5 No. 1, pp. 86-100. 
Panayiotou, N.A. and Stergiou, K.E. (2021), "A systematic literature review of lean six sigma adoption in European organizations", International Journal of Lean Six Sigma, Vol. 12 No. 2, pp. 264-292.

Signoretti, A. (2020), "Overcoming the barriers to the implementation of more efficient productive strategies in small enterprises", Employee Relations, Vol. 42 No. 1, pp. 149-165

Zhou, B. (2016) "Lean principles, practices, and impacts: a study on small and medium-sized enterprises (SMEs)”. Ann Oper Res 241, 457-474.

Acknowledgements: No external funding was received for this article. 